\title{
Bogomolny equations for the BPS Skyrme models with impurity
}

\section{t.T. Stẹpień}

The Pedagogical University of Cracow, ul. Podchorażych 2, 30-084 Kraków, Poland

E-mail: sfstepie@cyf-kr.edu.pl

ABSTRACT: We show that the BPS Skyrme model, as well as its $(2+1)$ dimensional baby version (restricted), can be coupled with an impurity in the BPS preserving manner. The corresponding Bogomolny equations are derived.

Keywords: Integrable Field Theories, Solitons Monopoles and Instantons, Effective Field Theories, Nonperturbative Effects

ARXIV EPRINT: 1912.10173 


\section{Contents}

1 Introduction 1

2 The concept of strong necessary conditions - a brief description 3

3 The derivation of the BPS equation for two models with impurity 4

3.1 The case of restricted baby BPS Skyrme model with impurity 4

3.2 The case of BPS Skyrme model with impurity 5

$\begin{array}{llr}4 & \text { Summary } & 7\end{array}$

\section{Introduction}

Addition of an impurity i.e., a non-dynamical background field, physically represents a nontrivial medium, in which a given field theory is immersed. Such medium effects may be due to local non-homogeneities as well as global dependence on external parameters. Especially, it is of some relevance for realistic solitonic systems, where typically a soliton exists not as an isolated object surrounded by vacuum, but, on the contrary, it exists in a presence of other solitons. Hence, an impurity can be also regarded as an averaged (integrated) impact of other solitons or as a frozen set of solitons (spectators). Another interesting impact of impurity, concerns vortex rings. They are such three-dimensional structures, where core is a closed loop with vorticity around it. Vortex rings can be produced alsoamong others, when impurity travels with a speed bigger than the speed of sound of the backround $[1,2]$.

A very important class of solitonic models are the so-called BPS theories [2-6]. Such a model possesses topologically nontrivial solutions saturating a pertinent topological lower bound on the energy. Because of that, the solutions are stable and follow from so-called Bogomolny equations, which are of lower order than the full Lagrange-Euler equations. Therefore, these models are much easier to solve than a generic model, and provide us a very important mathematical insight, especially in the case of higher dimensional topological solitons. Here let us mention the Abelian Higgs model at the critical coupling, 't Hooft-Polyakov monopole or Yang-Mills instantons as the most prominent examples. BPS property indicates for a given model that there is possible to derive Bogomolny equations for this model - such equations were derived for restricted BPS baby Skyrme model, [7, 8], for gauged restricted BPS baby Skyrme model, [9]. Some aspects of relations between supersymmetry and Bogomolny equations are considered in [10-12]. In [13, 15], higher derivative field-theoretical models (among others, generalized Skyrme models) and BPS states especially in the supersymmetric setups, were investigated, and various Bogomolny equations for these models were derived. Next, in [14] higher derivative corrections to supersymmetric realizations in the off-shell superfield formalism (without the problem of the 
auxiliary field) were constructed. A supersymmetric extension of the Skyrme term without the problem of the auxiliary field, was constructed in [18]. Some solitons and instantons for supersymmetric Skyrme model were found in [16] (the first pure Skyrme instanton had been presented in [17]).

Usually, coupling of an impurity to an arbitrary BPS model, breaks the BPS property completely, see for example [19]-[23]. Physically it means that static solitons, which in the original BPS theory do not interact with each other, begin to feel an attractive or repulsive force due to the presence of the impurity. In a consequence, the moduli space of energetically equivalent solutions (in the fixed topological sector) disappears as the solitons have energetically preferred positions (basically fixing the mutual distance). This has a nontrivial impact on solvability of the model (no Bogomolny equations) as well as its static and dynamical properties. Some vortex solutions in presence of magnetic impurity, were found in [24].

It has been recently found that there it is possible to couple an impurity to a BPS model, in such a way that half of the BPS property remains preserved [25, 26]. It means that half of originally BPS solitons keep this feature also in the presence of impurity [26, 27]. Hence, for these solutions there is some well defined moduli space. This was originally applied to $(1+1)$ dimensional models with kinks and to some $(2+1)$ planar solitons models [28].

A theory, where inclusion of impurities seems to be of great importance, is the Skyrme model [29-31]. This is one of the most popular effective theories of the low energy QCD describing non-perturbative excitations i.e., baryons and atomic nuclei as coherent solutions in the "mesonic fluid", that is (semiclassically quantized) solitons in a theory with pionic degrees of freedom [32-34] (fields in the Lagrangian). Furthermore, the Skyrme model is considered as relevant for description of nuclear matter at high density, where in-medium effects must nontrivially deform the theory. For our purposes it is important to note that at high density regime (relevant for in-medium/impurities effects), the generalized Skyrme model approaches a BPS theory, known as the BPS Skyrme model [35]. This limit of the generalised Skyrme model is considered to be crucial for description of (the inner core of) neutron stars [36, 37]. Hence, an obvious question arrises, whether $(3+1)$ dimensional BPS Skyrme model can be coupled with an impurity in a self-duality preserving manner. In [42] some class of models for static magnetic skyrmions, with presence of the DzyaloshinskiiMoriya interaction, is investigated, and it has been shown, that one can consider impurity as a non-abelian gauge field. BPS Skyrme model describes nucleons, when density is high and finite, for e.g. in a neutron star, where almost all the matter consists on neutrons, so as far as a physical situation, when impurity can appear in the case of BPS Skyrme model, is appearing of protons. However, the BPS Skyrme model is invariant with respect to isospin rotations. On the other hand, one can consider a situation, when breaking of isospin symmetry appears, and this scenario has been considered in [38]. Another source of impurity in nuclear matter, can be $\Lambda$ hyperon. An impact of such impurities on properties of nuclear matter, was considered among others, in [39] and [40]. Appearing of hypernuclei in nature, i.e. nuclei including among others $\Lambda$ hyperons, was verified experimentally in 1953 [41].

In this paper, we show that the BPS Skyrme model admits a coupling to an impurity such that the BPS property is kept unchanged. In particular we derive the Bogomolny 
equation for BPS family Skyrme models with a general form of the potential and with the impurity. We do it in a systematic way, namely, we apply the concept of strong necessary conditions (CSNC) presented in [43], and developed in [44-46, 50]. The application of the concept of CSNC, for deriving Bogomolny decomposition, was included in [47] (there were also described the relations between supersymmetry and Bogomolny decomposition derived by using CSNC).

This paper is organized, as follows. The next section contains a short description of the concept of strong necessary conditions. In the section 3, we derive Bogomolny equation for BPS family Skyrme models, by using this concept. In the section 4, we sum up the results, obtained in this paper.

\section{The concept of strong necessary conditions - a brief description}

The derivation of Bogomolny equations for some field theory systems in lower dimension, by using the CSNC method, was presented in [43, 45, 48-50]. It was also applied to baby Skyrme theories and their gauged version, in [8] and [9], correspondingly.

Here we briefly describe this method. As this is well-known fact, the Euler-Lagrange equations,

$$
F_{, u}-\frac{d}{d x} F_{, u, x}-\frac{d}{d y} F_{, u, y}=0,
$$

follow from $\delta \Phi[u]$, where $\Phi[u]$ is the functional

$$
\Phi[u]=\int_{E^{2}} F\left(u, u_{, x}, u_{, y}\right) d x d y .
$$

Instead of considering of (2.1), we consider strong necessary conditions, [43-45]

$$
\begin{aligned}
F_{, u} & =0, \\
F_{, u, x} & =0, \\
F_{, u, y} & =0,
\end{aligned}
$$

where $F_{, u} \equiv \frac{\partial F}{\partial u}$, etc.

Of course, the set of all solutions of the system of the equations (2.3)-(2.5), is a subset of the set of solutions of the Euler-Lagrange equation (2.1). However, if there exist the solutions of the equations (2.3)-(2.5), they are very often trivial solutions. So, in order to avoid this triviality, one can make gauge transformation of the functional (2.2)

$$
\Phi \rightarrow \Phi+\operatorname{Inv},
$$

where Inv is such functional that its local variation vanishes, with respect to $u(x, y)$ : $\delta \operatorname{Inv} \equiv 0$.

Owing to this feature, the Euler-Lagrange equations (2.1) and the Euler-Lagrange equations resulting from requiring of the extremum of $\Phi+I n v$, are equivalent. On the other hand, the strong necessary conditions (2.3)-(2.5) are not invariant with respect to the gauge transformation (2.6). Hence, now is a chance to obtain non-trivial solutions. Let us note that the order of the system of the partial differential equations, following from strong necessary conditions (2.3)-(2.5), is less than the order of Euler-Lagrange equations (2.1). 


\section{The derivation of the BPS equation for two models with impurity}

Now we show, how to apply the concept of strong necessary conditions in practice. We derive the Bogomolny equation for both models with impurity: BPS Skyrme model and restricted baby BPS Skyrme model.

\subsection{The case of restricted baby BPS Skyrme model with impurity}

According to the idea presented in the previous section, we make the gauge transformation of the density of the energy functional of the restricted baby BPS Skyrme model: $\mathcal{H} \longrightarrow \tilde{\mathcal{H}}$, however, in order to generalize the investigations, we consider

$$
\begin{aligned}
H=\int d x^{2} \mathcal{H}= & \int d^{2} x\left(f \cdot\left(\varepsilon^{m n} i \omega_{, m} \omega_{, n}^{*}\right)^{2}+V\right. \\
& \left.+\lambda_{1} \sigma\left(x^{k}\right)\left(\varepsilon^{m n} i \omega_{, m} \omega_{, n}^{*}\right)+\lambda_{2} \sigma\left(x^{k}\right) \sqrt{V}+\sigma^{2}\left(x^{k}\right)\right),
\end{aligned}
$$

where $f, V \in \mathcal{C}^{1}$ are some unspecified functions of $\omega, \omega^{*}$ and $k, m, n=1,2$. (For the sake of generality, we leave $f$ as a completely unspecified function, which correspond to a general target space geometry.) Next, we have to establish the general form of the density of the topological invariant $I_{1}=\int d^{2} x \mathcal{I}_{1}$, i.e. $\delta I_{1} \equiv 0$. It turns out that this density has the following form:

$$
\mathcal{I}_{1}=G_{1} \varepsilon^{m n} \omega_{, m} \omega_{, n}^{*},
$$

where $G_{1} \in \mathcal{C}^{1}$ is some arbitrary function of $\omega, \omega^{*}$.

The density of the gauged energy functional has the following form:

$$
\begin{aligned}
\tilde{\mathcal{H}}= & f \cdot\left(\varepsilon^{m n} i \omega_{, m} \omega_{, n}^{*}\right)^{2}+V \\
& +\lambda_{1} \sigma\left(x^{k}\right)\left(\varepsilon^{m n} i \omega_{, m} \omega_{, n}^{*}\right)+\lambda_{2} \sigma\left(x^{k}\right) \sqrt{V}+\sigma^{2}\left(x^{k}\right)+\sum_{r=1}^{3} \mathcal{I}_{r},
\end{aligned}
$$

where $f=f\left(\omega, \omega^{*}\right), V=V\left(\omega, \omega^{*}\right) \in \mathcal{C}^{1}, k, m, n=1,2$. $\mathcal{I}_{1}$ is given by (3.2), and $\mathcal{I}_{p+1}$ are the densities of so-called divergent invariants: $\mathcal{I}_{p+1}=D_{p} G_{p+1}, p=1,2 . G_{1}$ and $G_{p+1} \in \mathcal{C}^{1}$, are some functions of $\omega, \omega^{*}$, to be determined later.

The applying of strong necessary conditions, gives the so-called dual equations:

$$
\begin{aligned}
\tilde{\mathcal{H}}_{, \omega}: f_{, \omega} \cdot\left(\varepsilon^{m n} i \omega_{, m} \omega_{, n}^{*}\right)^{2}+V_{, \omega}+\lambda_{2} \frac{V_{, \omega}}{2 \sqrt{V}} \sigma+G_{1, \omega} \varepsilon^{m n} \omega_{, m} \omega_{, n}^{*}+\sum_{p=1}^{2} D_{p} G_{p+1, \omega}=0, \\
\tilde{\mathcal{H}}_{, \omega^{*}}: f_{, \omega^{*}} \cdot\left(\varepsilon^{m n} i \omega_{, m} \omega_{, n}^{*}\right)^{2}+V_{, \omega^{*}}+\lambda_{2} \frac{V_{, \omega^{*}}}{2 \sqrt{V}} \sigma+G_{1, \omega^{*}} \varepsilon^{m n} \omega_{, m} \omega_{, n}^{*}+\sum_{p=1}^{2} D_{p} G_{p+1, \omega^{*}}=0, \\
\tilde{\mathcal{H}}_{, \omega, r}: 2 f \cdot \varepsilon^{r j} i \omega_{, j}^{*}\left(\varepsilon^{m n} i \omega_{, m} \omega_{, n}^{*}\right)+\lambda_{1} \sigma \varepsilon^{r j} i \omega_{, j}^{*}+G_{1} \varepsilon^{r j} \omega_{, j}^{*}+G_{r+1, \omega}=0, \\
\tilde{\mathcal{H}}_{, \omega_{, r}^{*}}: 2 f \cdot \varepsilon^{j r} i \omega_{, j}\left(\varepsilon^{m n} i \omega_{, m} \omega_{, n}^{*}\right)+\lambda_{1} \sigma \varepsilon^{j r} i \omega_{, j}+G_{1} \varepsilon^{j r} \omega_{, j}+G_{r+1, \omega^{*}}=0 .
\end{aligned}
$$

Now we need to make the equations (3.4)-(3.7) self-consistent. At first, we want the equations (3.6)-(3.7) to be self-consistent. In this order, we put

$$
\begin{aligned}
2 i f \cdot\left(\varepsilon^{m n} i \omega_{, m} \omega_{, n}^{*}\right)+i \lambda_{1} \sigma+G_{1} & =0, \\
G_{r+1} & =\mathrm{const}, \quad r=1,2 .
\end{aligned}
$$


Hence, instead of two equations (3.6)-(3.7), we have one equation:

$$
2 i f \cdot\left(\varepsilon^{m n} i \omega_{, m} \omega_{, n}^{*}\right)+i \lambda_{1} \sigma+G_{1}=0,
$$

where $f=f\left(\omega, \omega^{*}\right) \in \mathcal{C}^{1}, G_{1}=G_{1}\left(\omega, \omega^{*}\right) \in \mathcal{C}^{1}$.

Now we have to eliminate from (3.4)-(3.5), all terms including the derivatives of the fields. In this order we use (3.8). Hence, we have two equations for the function $G_{1}$ and for the potential $V\left(\omega, \omega^{*}\right)$

$$
\begin{aligned}
-\frac{1}{4} \frac{f_{, \omega}\left(i \lambda_{1} \sigma+G_{1}\right)^{2}}{f^{2}}+V_{, \omega}+\frac{\lambda_{1} \sigma V_{, \omega}}{2 \sqrt{V}}+\frac{1}{2} \frac{G_{1, \omega}\left(i \lambda_{1} \sigma+G_{1}\right)}{f} & =0, \\
-\frac{1}{4} \frac{f_{, \omega^{*}}\left(i \lambda_{1} \sigma+G_{1}\right)^{2}}{f^{2}}+V_{, \omega^{*}}+\frac{\lambda_{1} \sigma V_{, \omega^{*}}}{2 \sqrt{V}}+\frac{1}{2} \frac{G_{1, \omega^{*}}\left(i \lambda_{1} \sigma+G_{1}\right) G_{1}}{f} & =0 .
\end{aligned}
$$

Their solution is:

$$
G_{1}=-i \lambda_{1} \sigma-\sqrt{f\left(-4 \lambda_{1} \sigma \sqrt{V}-4 V+C_{1}\right)}
$$

So we put $C_{1}=-C_{2}$ :

$$
G_{1}=-i \lambda_{1} \sigma-i \sqrt{f\left(4 \lambda_{1} \sigma \sqrt{V}+4 V+C_{2}\right)} .
$$

and we can eliminate $G_{1}$ from (3.9). Hence, we have the Bogomolny equation for the restricted baby BPS Skyrme model with impurity

$$
2 f \cdot\left(\varepsilon^{m n} i \omega_{, m} \omega_{, n}^{*}\right)=\sqrt{f\left(4 \lambda_{1} \sigma \sqrt{V}+4 V+C_{2}\right)} .
$$

\subsection{The case of BPS Skyrme model with impurity}

BPS Skyrme model with impurity is a mathematical generalization of the (restricted) baby BPS Skyrme model with impurity. The essence of this generalization is such that there in the hamiltonian appears the additional scalar field $\chi$, so the functions $f, V \in \mathcal{C}^{1}$ are now some unspecified functions of $\chi, \omega, \omega^{*}$, and each such function depends on three independent variables: $x, y, z$.

Again, we proceed according to the idea of the concept of strong necessary conditions. Namely, we make the gauge transformation of the density of the energy functional of the BPS Skyrme model: $\mathcal{H} \longrightarrow \tilde{\mathcal{H}}$, however, in order to generalize the investigations, we consider

$$
\begin{aligned}
H=\int d^{3} x \mathcal{H}= & \int d^{3} x\left(f \cdot\left(\varepsilon^{k m n} i \chi, k \omega_{, m} \omega_{, n}^{*}\right)^{2}+V\right. \\
& \left.+\lambda_{1} \sigma\left(x^{l}\right)\left(\varepsilon^{k m n} i \chi_{, k} \omega_{, m} \omega_{, n}^{*}\right)+\lambda_{2} \sigma\left(x^{l}\right) \sqrt{V}+\sigma^{2}\left(x^{k}\right)\right),
\end{aligned}
$$

where $f, V \in \mathcal{C}^{1}$ are some unspecified functions of $\chi, \omega, \omega^{*}$ and $k, l, m, n=1,2,3$ (as in the previous model, for the sake of generality, we assume that $f$ is some completely unspecified function, corresponding to a general target space geometry. The usual $S^{3}$ target space is obtained for $f=\frac{\sin ^{4} \chi}{\left(1+|\omega|^{2}\right)^{4}}$.) Next, we have to establish the general form of the density of 
the topological invariant $I_{1}=\int d^{3} x \mathcal{I}_{1}$, i.e. $\delta I_{1} \equiv 0$. It turns out that this density has the following form:

$$
\mathcal{I}_{1}=G_{1} \varepsilon^{k m n} \chi_{, k} \omega_{, m} \omega_{, n}^{*}
$$

where $G_{1} \in \mathcal{C}^{1}$ is some arbitrary function of $\chi, \omega, \omega^{*}$.

The density of the gauged energy functional has the following form:

$$
\begin{aligned}
\tilde{\mathcal{H}}= & f \cdot\left(\varepsilon^{k m n} i \chi_{, k} \omega_{, m} \omega_{, n}^{*}\right)^{2}+V \\
& +\lambda_{1} \sigma\left(x^{l}\right)\left(\varepsilon^{k m n} i \chi_{, k} \omega_{, m} \omega_{, n}^{*}\right)+\lambda_{2} \sigma\left(x^{l}\right) \sqrt{V}+\sigma^{2}\left(x^{l}\right)+\sum_{r=1}^{4} \mathcal{I}_{r}
\end{aligned}
$$

where $f=f\left(\chi, \omega, \omega^{*}\right), V=V\left(\chi, \omega, \omega^{*}\right) \in \mathcal{C}^{1}, k, l, m, n=1,2,3 . \mathcal{I}_{1}$ is given by (3.2), and $\mathcal{I}_{p+1}$ are the densities of so-called divergent invariants: $\mathcal{I}_{p+1}=D_{p} G_{p+1}, p=1, \ldots, 3 . G_{1}$, and $G_{p+1} \in \mathcal{C}^{1}$, are some functions of $\chi, \omega, \omega^{*}$, to be determined later.

The applying of strong necessary conditions, gives the so-called dual equations:

$$
\begin{aligned}
& \tilde{\mathcal{H}}_{, \chi}: f_{, \chi} \cdot\left(\varepsilon^{k m n} i \chi, k \omega_{, m} \omega_{, n}^{*}\right)^{2} \\
& +V_{, \chi}+\lambda_{2} \sigma \frac{V_{, \chi}}{2 \sqrt{V}}+G_{1, \chi} \varepsilon^{k m n} \chi_{, k} \omega_{, m} \omega_{, n}^{*}+\sum_{p=1}^{3} D_{p} G_{p+1, \chi}=0, \\
& \tilde{\mathcal{H}}_{, \omega}: f_{, \omega} \cdot\left(\varepsilon^{k m n} i \chi_{, k} \omega_{, m} \omega_{, n}^{*}\right)^{2} \\
& +V_{, \omega}+\lambda_{2} \sigma \frac{V_{, \omega}}{2 \sqrt{V}}+G_{1, \omega} \varepsilon^{k m n} \chi_{, k} \omega_{, m} \omega_{, n}^{*}+\sum_{p=1}^{3} D_{p} G_{p+1, \omega}=0, \\
& \tilde{\mathcal{H}}_{, \omega^{*}}: f_{, \omega^{*}} \cdot\left(\varepsilon^{k m n} i \chi_{, k} \omega_{, m} \omega_{, n}^{*}\right)^{2} \\
& +V_{, \omega^{*}}+\lambda_{2} \sigma \frac{V_{, \omega^{*}}}{2 \sqrt{V}}+G_{1, \omega^{*}} \varepsilon^{k m n} \chi_{, k} \omega_{, m} \omega_{, n}^{*}+\sum_{p=1}^{3} D_{p} G_{p+1, \omega^{*}}=0, \\
& \tilde{\mathcal{H}}_{, \chi_{, r}}: 2 f \cdot \varepsilon^{r s j} i \omega_{, s} \omega_{, j}^{*}\left(\varepsilon^{k m n} i \chi_{, k} \omega_{, m} \omega_{, n}^{*}\right) \\
& +\sigma \varepsilon^{r s j} i \omega_{, s} \omega_{, j}^{*}+G_{1} \varepsilon^{r s j} \omega_{, s} \omega_{, j}^{*}+G_{r+1, \chi}=0, \\
& \tilde{\mathcal{H}}_{, \omega, r}: 2 f \cdot \varepsilon^{s r j} i \chi_{, s} \omega_{, j}^{*}\left(\varepsilon^{k m n} i \chi_{, k} \omega_{, m} \omega_{, n}^{*}\right) \\
& +\sigma \varepsilon^{s r j} i \chi{ }_{, s} \omega_{, j}^{*}+G_{1} \varepsilon^{s r j} \chi_{, s} \omega_{, j}^{*}+G_{r+1, \omega}=0, \\
& \tilde{\mathcal{H}}_{, \omega_{, r}^{*}}: 2 f \cdot \varepsilon^{s j r} i \chi_{, s} \omega_{, j}\left(\varepsilon^{k m n} i \chi_{, k} \omega_{, m} \omega_{, n}^{*}\right) \\
& +\sigma \varepsilon^{s j r} i \chi_{, s} \omega_{, j}+G_{1} \varepsilon^{s j r} \chi_{, s} \omega_{, j}+G_{r+1, \omega^{*}}=0 .
\end{aligned}
$$

Now we need to make the equations (3.17)-(3.22), self-consistent. At first, analogically to the previous model, we want the equations (3.20)-(3.22) to be self-consistent. In this order, we put

$$
\begin{aligned}
& 2 i f \cdot\left(\varepsilon^{k m n} i \chi, k \omega_{, m} \omega_{, n}^{*}\right)+i \lambda_{1} \sigma+G_{1}=0, \\
& G_{r+1}=\text { const }, \quad r=1,2,3 .
\end{aligned}
$$

Hence, instead of three equations (3.20)-(3.22), we have one equation:

$$
2 i f \cdot\left(\varepsilon^{k m n} i \chi_{, k} \omega_{, m} \omega_{, n}^{*}\right)+i \lambda_{1} \sigma+G_{1}=0
$$


where $f=f\left(\chi, \omega, \omega^{*}\right) \in \mathcal{C}^{1}, G_{1}=G_{1}\left(\chi, \omega, \omega^{*}\right) \in \mathcal{C}^{1}$. Next, we again eliminate from (3.17)(3.19), all terms including the derivatives of the fields, by using (3.8). Hence, we have three equations for the function $G_{1}$ and for the potential $V\left(\chi, \omega, \omega^{*}\right)$

$$
\begin{array}{r}
-\frac{1}{4} \frac{f_{, \chi}\left(i \lambda_{1} \sigma+G_{1}\right)^{2}}{f^{2}}+V_{, \chi}+\frac{\lambda_{1} \sigma V_{, \chi}}{2 \sqrt{V}}+\frac{1}{2} \frac{G_{1, \chi}\left(i \lambda_{1} \sigma+G_{1}\right)}{f}=0, \\
-\frac{1}{4} \frac{f_{, \omega}\left(i \lambda_{1} \sigma+G_{1}\right)^{2}}{f^{2}}+V_{, \omega}+\frac{\lambda_{1} \sigma V_{, \omega}}{2 \sqrt{V}}+\frac{1}{2} \frac{G_{1, \omega}\left(i \lambda_{1} \sigma+G_{1}\right)}{f}=0, \\
-\frac{1}{4} \frac{f_{, \omega^{*}}\left(i \lambda_{1} \sigma+G_{1}\right)^{2}}{f^{2}}+V_{, \omega^{*}}+\frac{\lambda_{1} \sigma V_{, \omega^{*}}}{2 \sqrt{V}}+\frac{1}{2} \frac{G_{1, \omega^{*}}\left(i \lambda_{1} \sigma+G_{1}\right)}{f}=0 .
\end{array}
$$

Their solution is analogical to the function $G_{1}$ in the case of baby BPS Skyrme model with impurity:

$$
G_{1}=-i \lambda_{1} \sigma-i \sqrt{f\left(4 \lambda_{1} \sigma \sqrt{V}+4 V+C_{2}\right)},
$$

where instead of the integration constant $C_{1}$, again the constant $C_{2}$ appears $\left(C_{1}=-C_{2}\right)$.

Thus, we can again eliminate $G_{1}$ from (3.24). Then, the Bogomolny equation for the BPS Skyrme model with impurity has the form:

$$
2 f \cdot\left(\varepsilon^{k m n} i \chi_{, k} \omega_{, m} \omega_{, n}^{*}\right)=\sqrt{f\left(4 \lambda_{1} \sigma \sqrt{V}+4 V+C_{2}\right)} .
$$

\section{Summary}

In this paper the Bogomolny equations for the BPS Skyrme model and restricted baby BPS Skyrme model, with impurity terms and with the general forms of the potentials $V$, were derived, by using the concept of strong necessary conditions method. As one can see, in the absence of the impurity term $(\sigma=0)$, the found Bogomolny equations and conditions for the functions $G_{1}$ become into their versions for BPS Skyrme model [51], and restricted BPS baby Skyrme model without impurity terms [8] (in the last case, $C_{2}=0$ ). Analogically, as in [51] (where the impurity term was absent), we re-derive the Bogomolny equation for $C_{2}=0$ (obviously, if a proper choice of the function $f$ is done) and a non-Bogomolny equation for $C_{2}>0$. In the case, when $C_{2}>0$, we have an analogical situation to that one without impurity term, when there is a coincidence with non-zero pressure equation for the BPS Skyrme model [52].

This result provides the first example of BPS preserving impurity model in $(3+1)$ dimension. The (non-)Bogomolny equations were derived for general form of the potential $V$, when $V=V\left(\chi, \omega, \omega^{*}\right)$, and in this case we have isospin symmetry breaking [38]. So, in such situation, one can expect the situation, when impurity appears, because of protons.

Computational resources. Some part of computations was carried out by using Maple Waterloo Software, owing to the financial support, provided by The Pedagogical University of Cracow, within a research project (the leader of this project: Dr. K. Rajchel).

\section{Acknowledgments}

The author thanks to Prof. A. Wereszczyński for his valuable remarks. 
Open Access. This article is distributed under the terms of the Creative Commons Attribution License (CC-BY 4.0), which permits any use, distribution and reproduction in any medium, provided the original author(s) and source are credited.

\section{References}

[1] A.S. Rodrigues et al., Spinor Bose-Einstein condensate flow past an obstacle, Phys. Rev. A 79 (2009) 043603.

[2] A. Saxena, P.G. Kevrekidis and J. Cuevas-Maraver, Nonlinearity and topology, in Emerging frontiers in nonlinear science, P.G. Kevrekidis, J. Cuevas-Maraver and A. Saxena eds., Springer, Cham, Switzerland (2020), pg. 25.

[3] N. Manton and P. Sutcliffe, Topological solitons, Cambridge University Press, Cambridge, U.K. (2004).

[4] A. Ludu, Nonlinear waves and solitons on contours and closed surfaces, Springer, Berlin, Heidelberg, Germany (2012).

[5] Y.M. Shnir, Topological and non-topological solitons in scalar field theories, Cambridge University Press, Cambridge, U.K. (2018).

[6] N.S. Manton, Nonlinearity, geometry and field theory solitons, in Emerging frontiers in nonlinear science, P.G. Kevrekidis, J. Cuevas-Maraver and A. Saxena eds., Springer, Cham, Switzerland (2020), pg. 205.

[7] C. Adam, T. Romańczukiewicz, J. Sánchez-Guillén and A. Wereszczyński, Investigation of restricted baby Skyrme models, Phys. Rev. D 81 (2010) 085007 [arXiv:1002.0851] [INSPIRE].

[8] Ł.T. Stȩpień, On Bogomolny decompositions for the baby Skyrme models, in $31^{\text {st }}$ workshop on geometric methods in physics, Bialowieza, Poland, 24-30 June 2012, P. Kielanowski, S.T. Ali et al. eds., Birkhäuser, Basel, Switzerland (2013), pg. 229. [arXiv:1204.6194] [INSPIRE].

[9] Ł.T. Stȩpień, The existence of Bogomolny decompositions for gauged $O(3)$ nonlinear "sigma" model and for gauged baby Skyrme models, Acta Phys. Polon. B 46 (2015) 999 [arXiv: 1205.1017] [INSPIRE].

[10] Z. Hlousek and D. Spector, Why topological charges imply extended supersymmetry, Nucl. Phys. B 370 (1992) 143 [INSPIRE].

[11] Z. Hlousek and D. Spector, Bogomolny explained, Nucl. Phys. B 397 (1993) 173 [inSPIRE].

[12] B. Damski, Supersymmetry and Bogomolnyi equations in the Maxwell Chern-Simons systems, Acta Phys. Polon. B 31 (2000) 637 [hep-th/0001022] [INSPIRE].

[13] M. Nitta and S. Sasaki, BPS states in supersymmetric chiral models with higher derivative terms, Phys. Rev. D 90 (2014) 105001 [arXiv:1406.7647] [INSPIRE].

[14] M. Nitta and S. Sasaki, Higher derivative corrections to manifestly supersymmetric nonlinear realizations, Phys. Rev. D 90 (2014) 105002 [arXiv:1408.4210] [INSPIRE].

[15] M. Nitta and S. Sasaki, Classifying BPS states in supersymmetric gauge theories coupled to higher derivative chiral models, Phys. Rev. D 91 (2015) 125025 [arXiv:1504.08123] [INSPIRE].

[16] S.B. Gudnason, M. Nitta and S. Sasaki, Topological solitons in the supersymmetric Skyrme model, JHEP 01 (2017) 014 [arXiv:1608.03526] [INSPIRE]. 
[17] J.M. Speight, A pure Skyrme instanton, Phys. Lett. B 659 (2008) 429 [hep-th/0703198] [INSPIRE].

[18] S.B. Gudnason, M. Nitta and S. Sasaki, A supersymmetric Skyrme model, JHEP 02 (2016) 074 [arXiv: 1512.07557] [INSPIRE].

[19] F. Zhang, Y.S. Kivshar, B.A. Malomed and L. Vázquez, Kink capture by a local impurity in the sine-Gordon model, Phys. Lett. A 159 (1991) 318.

[20] Z. Fei, L. Vazquez and Y.S. Kivshar, Resonant kink impurity interactions in the $\phi^{4}$ model, Phys. Rev. A 46 (1992) 5214 [InSPIRE].

[21] T. Dobrowolski, Kink production in the presence of impurities, Phys. Rev. E 65 (2002) 036136 .

[22] B. Piette and W.J. Zakrzewski, Scattering of sine-Gordon breathers on a potential well, Phys. Rev. E 79 (2009) 046603.

[23] S.W. Goatham, L.E. Mannering, R. Hann and S. Krusch, Dynamics of multi-kinks in the presence of wells and barriers, Acta Phys. Polon. B 42 (2011) 2087 [arXiv:1007.2641] [INSPIRE].

[24] J. Ashcroft and S. Krusch, Vortices and magnetic impurities, Phys. Rev. D 101 (2020) 025004 [arXiv: 1808.07441] [INSPIRE].

[25] C. Adam and A. Wereszczyński, BPS property and its breaking in $1+1$ dimensions, Phys. Rev. D 98 (2018) 116001 [arXiv: 1809.01667] [INSPIRE].

[26] C. Adam, T. Romańczukiewicz and A. Wereszczyński, The $\phi^{4}$ model with the BPS preserving defect, JHEP 03 (2019) 131 [arXiv:1812.04007] [INSPIRE].

[27] C. Adam, K. Oleś, J.M. Queiruga, T. Romańczukiewicz and A. Wereszczyński, Solvable self-dual impurity models, JHEP 07 (2019) 150 [arXiv: 1905.06080] [INSPIRE].

[28] C. Adam, J.M. Queiruga and A. Wereszczynski, BPS soliton-impurity models and supersymmetry, JHEP 07 (2019) 164 [arXiv: 1901.04501] [INSPIRE].

[29] T.H.R. Skyrme, A nonlinear field theory, Proc. Roy. Soc. Lond. A 260 (1961) 127 [INSPIRE].

[30] T.H.R. Skyrme, A unified field theory of mesons and baryons, Nucl. Phys. 31 (1962) 556 [INSPIRE].

[31] T.H.R. Skyrme, Kinks and the Dirac equation, J. Math. Phys. 12 (1971) 1735 [inSPIRE].

[32] G.S. Adkins, C.R. Nappi and E. Witten, Static properties of nucleons in the Skyrme model, Nucl. Phys. B 228 (1983) 552 [INSPIRE].

[33] O.V. Manko, N.S. Manton and S.W. Wood, Light nuclei as quantized skyrmions, Phys. Rev. C 76 (2007) 055203 [arXiv: 0707.0868] [INSPIRE].

[34] C.J. Houghton, N.S. Manton and P.M. Sutcliffe, Rational maps, monopoles and skyrmions, Nucl. Phys. B 510 (1998) 507 [hep-th/9705151] [InSPIRE].

[35] C. Adam, J. Sanchez-Guillen and A. Wereszczyński, A Skyrme-type proposal for baryonic matter, Phys. Lett. B 691 (2010) 105 [arXiv:1001.4544] [INSPIRE].

[36] C. Adam, C. Naya, J. Sanchez-Guillen, R. Vazquez and A. Wereszczyński, BPS skyrmions as neutron stars, Phys. Lett. B $\mathbf{7 4 2}$ (2015) 136 [arXiv:1407.3799] [INSPIRE].

[37] C. Naya, Neutron stars within the Skyrme model, Int. J. Mod. Phys. E 28 (2019) 1930006 [arXiv: 1910.01145] [INSPIRE]. 
[38] P. Klimas, Composite BPS skyrmions from an exact isospin symmetry breaking, Acta Phys. Polon. B 47 (2016) 2245 [arXiv: 1602.00908] [INSPIRE].

[39] J. Dabrowski, Hyperons in nuclear matter - an impurity problem, in Recent progress in many-body theories, J.G. Zabolitzky, M. de Llano, M. Fortes and J.W. Clark eds., Lect. Notes Phys. 142, Springer, Berlin, Heidelberg, Germany (1981), pg. 426.

[40] H. Tamura, Impurity nuclear physics, Eur. Phys. J. A 13 (2002) 181.

[41] M. Danysz and J. Pniewski, Delayed disintegration of a heavy nuclear fragment: I, Phil. Mag. 44 (1953) 348.

[42] B.J. Schroers, Gauged $\sigma$-models and magnetic skyrmions, SciPost Phys. 7 (2019) 030 [arXiv: 1905.06285] [INSPIRE].

[43] K. Sokalski, Instantons in anisotropic ferromagnets, Acta Phys. Polon. A 56 (1979) 571.

[44] K. Sokalski, T. Wietecha and Z. Lisowski, Variational approach to the Bäcklund transformations, Acta Phys. Polon. B 32 (2001) 17.

[45] K. Sokalski, T. Wietecha and Z. Lisowski, A concept of strong necessary condition in nonlinear field theory, Acta Phys. Polon. B 32 (2001) 2771 [INSPIRE].

[46] K. Sokalski, T. Wietecha and Z. Lisowski, Unified variational approach to the Bäcklund transformation and the Bogomolny decomposition, Int. J. Theor. Phys. Group Theor. Nonlin. Opt. NOVA 9 (2002) 331.

[47] K. Sokalski, Ł. Stȩpień and D. Sokalska, The existence of Bogomolny decomposition by means of strong necessary conditions, J. Phys. A 35 (2002) 6157.

[48] L. Stẹpień, Bogomolny decomposition in the context of the concept of strong necessary conditions (in Polish), Ph.D. dissertation, Marian Smoluchowski Institute of Physics, Department of Mathematics, Physics and Astronomy, Jagiellonian University, Krakow, Poland (2003).

[49] Ł. Stȩpień, D. Sokalska and K. Sokalski, The Bogomolny decomposition for systems of two generalized nonlinear partial differential equations of the second order, J. Nonlin. Math. Phys. 16 (2009) 25.

[50] C. Adam and F. Santamaria, The first-order Euler-Lagrange equations and some of their uses, JHEP 12 (2016) 047 [arXiv: 1609.02154] [INSPIRE].

[51] Ł.T. Stȩpień, Bogomolny equation for the BPS Skyrme model from strong necessary conditions, J. Phys. A 49 (2016) 175202 [arXiv:1512.01231] [INSPIRE].

[52] C. Adam, C. Naya, J. Sanchez-Guillen, J.M. Speight and A. Wereszczyński, Thermodynamics of the BPS Skyrme model, Phys. Rev. D 90 (2014) 045003 [arXiv:1405.2927] [InSPIRE]. 